making such shots. It is frequently of great advantage to run slow-motion films backwards. In such circumstances many points are obvious which can scarcely be noticed in a normal forward run. Illustrative excerpts of films of a potato digger and a disk-plough in action were shown.

The 'high light' of the afternoon was a speeded-up film of ploughing, made at Askham Bryan, to teach the best methods of laying out and ploughing a field so that idle running of plough and tractor is reduced to a minimum. A suitable field on Teesside overlooked by a eliff was the site, and the whole operation of setting out and ploughing was photographed from the top of the cliff, one picture being taken every second. In this way it was possible to compress two days ploughing into a film which could be shown at the normal rate in 40 minutes. The effect, as well as being very instructive, was amusing, as the tractor and plough appeared to move at about 50 m.p.h.

In addition to these direct methods of employing photography, two uses of the photographic emulsion at Rothamsted were touched upon: spectrographic analysis, both qualitative and quantitative, and $\mathrm{X}$-ray diffraction work on clay minerals, in both of which the sensitive emulsion is used as a recording medium. Standard equipment is used for both-a Hilger medium quartz spectrograph and a Cambridge cylindrical powder camera of $28 \mathrm{~mm}$. radius.

The spectrographic work includes the analysis of soils and plants for such elements as calcium, potassium, sodium, manganese and magnesium, and the detection and estimation of trace elements such as copper and boron and, in one or two recent instances, zinc, lead and chromium in toxic soils. Elements of the first group are estimated from lines excited in the Lundegärdh burner, and calibration curves are plotted with the ratio of line density to background density as ordinate and logarithms of the concentration as abscissæ. Elements of the second group are estimated from lines excited in the carbon and copper are, using suitable internal standards; for example, tin for boron, the intensity of the tin line at $2496 \mathrm{~A}$. being compared with the boron line at $2498 \mathrm{~A}$.

The X-ray work is indispensable for the identification of minerals found in soil colloids and clays. The electron microscope will undoubtedly help greatly in this work, but as yet, insufficient data are available for the best use to be made of its possibilities. As a general rule, powder diagrams. of clay minerals are poor because of the large scattering near the central beam, and aggregate diagrams must be used instead. However, the newer technique of replacing the air in the camera by hydrogen so cuts down the scattering as to make the former more valuable, particularly when obtained from an aggregate.

In the short discussion following the papers, Prof. Yule Bogue raised the question of the limitation of the electron microscope for the examination of biological material by the necessity for working in a vacuum. Dr. Crook, replying, pointed out that the vacuum is only one of the limitations, since the electron-scattering power of the oxygen atoms of the water which constitutes so large a proportion of living material is much greater than the scattering power of the carbon and nitrogen atoms which are the chief 'organic' constituents, and that we can at best hope for an image rather like a radiograph if it becomes possible to use wet material (say, by a freezing technique).

\section{NUCLEAR ENERGY-LEVELS}

THE following is a summary of a paper by $P$. Comparat* which came into the writer's hands by an unorthodox but topical procedure. Printed at Lyons in 1942 , it was brought from France by an officer of the Free French forces in 1943. It then found its way into the hands of Prof. D. Saurat, of the French Department of King's College, London, who presented it to the College library. The work described therein was carried out at the Faculty of Sciences, Lyons, initially under the direction of Prof. J. Thibaud. The research occupied a period of four years, full time being devoted to the problem.

Introduction. The first demonstration of the transmutation of nitrogen by rapid neutrons was that of Feather in 1932, using a cloud expansion chamber. The suggested reaction was

$$
{ }^{12} \mathrm{~N}+n \rightarrow{ }^{4} \mathrm{He}+{ }^{11} \mathrm{~B}+Q,
$$

where $Q$ was negative, thus corresponding to an endoenergetic reaction. Subsequent workers, for example, Chadwick and Goldhaber, showed in 1935 with an ionization chamber and a linear amplifier that exo-energetic disintegration also occurred in this reaction. About the same time, Kurie demonstrated that an alternative reaction occurred in which protons were emitted instead of $\alpha$-particles.

The aim of Comparat's research was to measure the number of disintegrations at various energies of disintegration and to see if the magnitude and distribution of the energy-levels agreed with the current theory. By 1.936 the discord between theory and experiment was considerable; the existing theory,

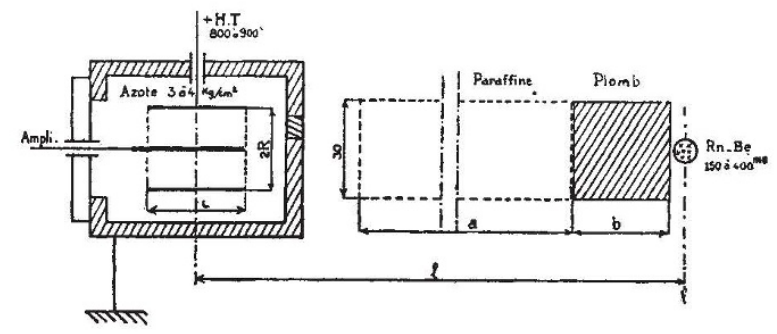

Fig. 1.

based on the two-body concept of the interaction of the incident neutron with the ${ }^{14} \mathrm{~N}$ nucleus, predicted a few nuclear levels of considerable width. On the contrary, experiment showed numerous sharp levels. Again, theory predicted identical values for the diffusion and capture cross-sections of slow neutrons, whereas experiment showed a large ratio.

At about this time, Bohr introduced the concept of nuclear transmutation according to which the incident neutron amalgamates with the entire nucleus to form an intermediate nucleus (in this case ${ }^{15} \mathrm{~N}$ ) of appreciable duration. The intermediate nucleus afterwards disintegrates, for it is in an excited state, with the emission of a heavy particle, often accompanied by $\gamma$-radiation. In the special case of nitrogen bombarded by neutrons and boron bombarded by $\alpha$-particles, the whole process is reversible, thus:

* "Disintegration of Nitrogen by Fast Neutrons; Distribution of the Energy Levels of $\mathrm{N}^{15}$ ". By P. Comparat. Pp. 96. (Lyon, 1942.) 


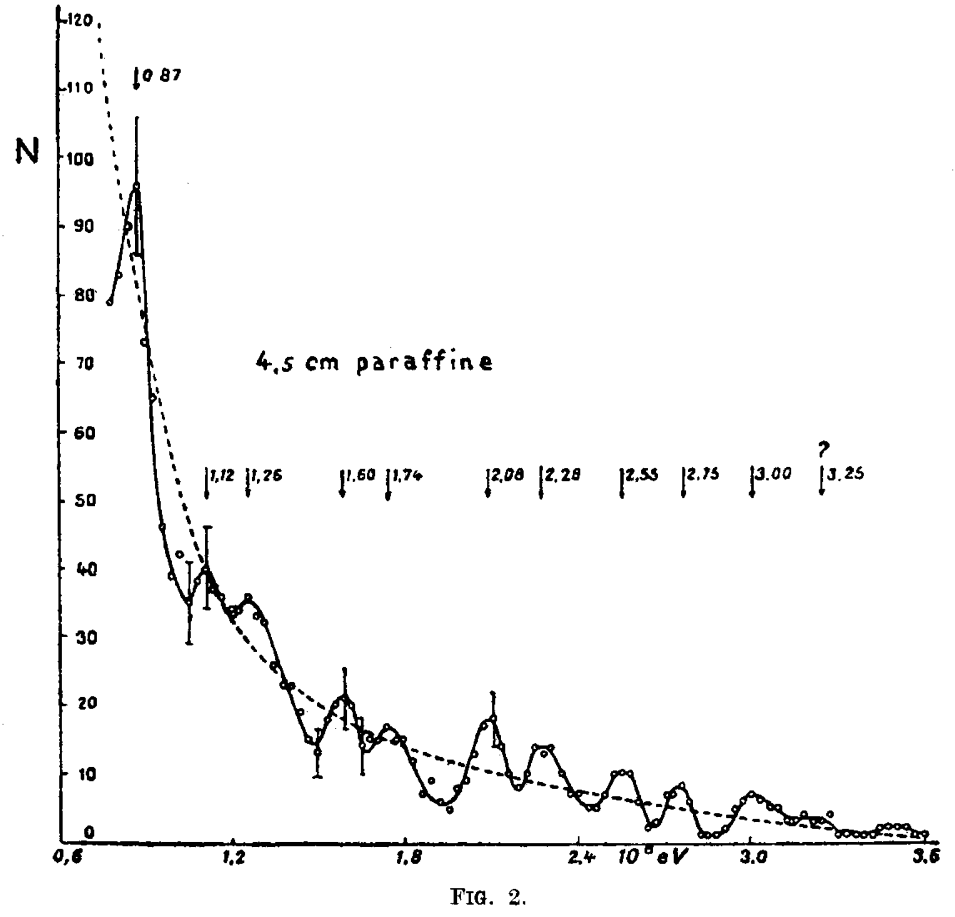

${ }^{14} \mathrm{~N}+{ }^{1} n \rightarrow{ }^{15} \mathrm{~N} \rightarrow{ }^{11} \mathrm{~B}+{ }^{4} \mathrm{He}$

and also

$$
{ }^{11} \mathrm{~B}+{ }^{4} \mathrm{He} \rightarrow{ }^{15} \mathrm{~N} \rightarrow{ }^{14} \mathrm{~N}+{ }^{1} n
$$

Hence the same resonance levels of ${ }^{15} \mathrm{~N}$ should be registered in both cases, although the ${ }^{15} \mathrm{~N}$ has been prepared by two quite different processes.

By 1937, several determinations had been made of the values of the excited levels of ${ }^{15} \mathrm{~N}$. Wilhelmy had demonstrated the existence of at least five distinct levels for reaction (1), while Maurer had recorded eight levels for reaction (2). Agreement was found between the two workers with four of the energy values. Of the remainder, two further values were assigned to ${ }^{15} \mathrm{~N}$, but the other two were attributed to the excited isotope $1 \mathbb{N}$, formed from the boron isotope ${ }^{10} \mathrm{~B}$, which was present in the experiment. Szalay confirmed Maurer's six levels for ${ }^{15} \mathrm{~N}$ and added one more. Finally, Ortner and Protowinsky found a total of twelve groups of particles emitted by ${ }^{15} \mathrm{~N}$ in the energy range $1-7 \mathrm{MV}$.

Experimental arrangement. A diagram of the experimental arrangement is shown in Fig. 1. The principle of the method consists in the use of a proportional counter connected to a linear amplifier and an oscillograph to record the total energy of disintegration of the $\alpha$-particle together with that of the residual recoiling nucleus ${ }^{11 B}$.

The counter has a cylindrical anode of about $25 \mathrm{~mm}$. diameter ; this was positively charged. The negative electrode was an axial filament $0 \cdot 5-1 \mathrm{~mm}$. in diameter. The pressure of nitrogen filling the counter was 4-5 atmosphere. At this pressure not only is the ionization increased but also the range of the disintegration products is sufficiently reduced to ensure their being entirely contained in the counter. Zinc was chosen as the metal for the outer electrode on the grounds of ease of purification; the total effective volume of the counter was $15-20$ c.c. The potential difference between anode and cathode was about 900 volts and the counter filament was connected to the grid of the first amplifying valve.

The source of neutrons was the usual radon - beryllium mixture of strength 150-400 millicuries. Difficulties arising from the intense $\gamma$ radiation were reduced to a minimum by the insertion of absorbing blocks of lead. For details of the linear amplifier, reference is made to an earlier publication of Thibaud, Cartan and Comparat (1938). Special precautions were taken to maintain constant conditions of amplification; for example, changes in resistance were prevented by keeping the apparatus to within $5^{\circ}-10^{\circ} \mathrm{C}$. by thermostat. The sensitivity and reliability of the instrument was checked at frequent intervals with a standard polonium source. As a further check, the constancy of amplification was tested by measurement of the heights of the peaks observed on the oscillograph when a weak A.c. potential was applied to the ionization chamber. The oscillograph was of the Dubois electromagnetic type and this suffered from some zero displacement.

Measurements. The final results were expressed in the form of curves with $N=f\left(E_{a+B}\right)$, where $N$ is the number of peaks corresponding to a total energy of the disintegration products $E_{a+B}$. Apart from the error arising from statistical fluctuations, which could be readily estimated, the chief source of uncertainty lay in the determination of the exact heights of the peaks. These heights varied from $10 \mathrm{~mm}$. to $50 \mathrm{~mm}$. and it was estimated that measurements

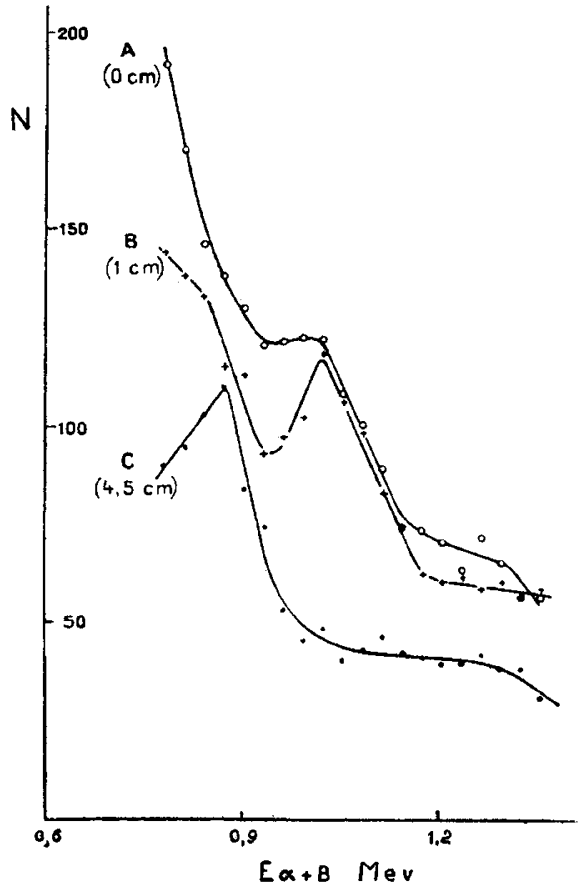

FIG. 3. 
could be made to within $0.25-0.5 \mathrm{~mm}$. Hence for smaller energies, corresponding to less than I MV., the maximum error was about 5 per cent, while it was correspondingly smaller at larger energies. With an intense $\gamma$-ray background, however, the zero band is widened considerably and the error may rise to 3-4 mm. The effect is strikingly demonstrated using $\alpha$-particles from a polonium source. In the presence of $\gamma$-radiation, a typical $\alpha$-ray peak is reduced in height by more than 50 per cent and increased in breadth by a factor of three or four times. Hence in deciding the reality of the existence of a flattened resonance group, some procedure such as the taking of several independent recordings was carried out to see if the point of inflexion was repeated.

Several sets of measurements were made with the same counter but under somewhat different experimental conditions. Some correspond to a layer of paraffin wax, 0-10 cm. thick, interposed between the source and the ionization chamber; others were made with no wax and only a sufficiently thick layer of lead to absorb most of the $\gamma$-radiation. As a control on the effect of the thermal neutrons, a thin layer of cadmium (0-5 mm.) could be inserted, and as a check on the behaviour of the ionization chamber, this could be filled with hydrogen instead of nitrogen. Typical of the measurements made were the following : (a) Ten recordings of 1 hour each made with different thicknesses of wax and with neutrons in the energy range $0 \cdot 5-1.8 \mathrm{MV}$. (b) Three recordings of 4 hours each with three different thicknesses of wax -energy range of neutrons $0 \cdot 75-3 \cdot 6 \mathrm{MV}$., and so on.

Results. Some of the results obtained are shown in Figs. 2 and 3. In Fig. 2, the energy range of the incident neutrons was $0 \cdot 75-3 \cdot 6 \mathrm{MV}$. with a thickness of wax of $4.5 \mathrm{~cm}$. The general behaviour, which is quite typical, shows a decrease of intensity with energy, together with distinct groups superposed on the smoothed curve, for example, at energies of 0.87 , $1 \cdot 12,1 \cdot 26,1 \cdot 60,1 \cdot 74,2 \cdot 08,2 \cdot 28,2 \cdot 55,2 \cdot 75(3 \cdot 00)$ and $(3 \cdot 25) \mathrm{MV}$. The figures in brackets are considered to be less well established than the others.

The effect of varying the thickness of the wax while maintaining other conditions constant is shown in Fig. 3 for the energy range $0 \cdot 75-1 \cdot 3 \mathrm{MV}$. Curve $A$ was taken with wax absent, while curves $B$ and $C$ corresponded to thicknesses of wax of $1 \mathrm{~cm}$. and $4.5 \mathrm{~cm}$. respectively. It is noted that some maxima visible with one thickness of wax vanish with another thickness, while other maxima seem little affected. In particular, on passing from $A$ to $B$, considerable diminution is observed in the number of low-energy particles, while on passing from $B$ to $C$ there is a further reduction in the number of low-energy particles; also a pronounced maximum appears at $0.87 \mathrm{MV}$., that previously at 1.02 MV. having disappeared. Further curves obtained for energies greater than 1.4 MV. give maxima the appearance and position of which are little affected by the presence and thickness of the wax.

Throughout this work, control experiments carried out with hydrogen replacing nitrogen in the ionization chamber gave a smooth curve with complete absence of peaks.

In all, thirty-two distinct groups of energies in the range $0 \cdot 5-7 \mathrm{MV}$. were established as follows : $0 \cdot 60$, $0 \cdot 75,0 \cdot 90,1 \cdot 05,1 \cdot 25,1 \cdot 40,1 \cdot 60,1 \cdot 75,2 \cdot 05,2 \cdot 25$, $(2 \cdot 42), 2 \cdot 6,2 \cdot 78,3 \cdot 03,3 \cdot 18,3 \cdot 33,3 \cdot 46,3 \cdot 63,3 \cdot 76$, $4 \cdot 02,4 \cdot 20,4 \cdot 36,4 \cdot 66,4 \cdot 82,4 \cdot 98,5 \cdot 27,5 \cdot 58,(6 \cdot 04)$, $(6 \cdot 22),(6 \cdot 41),(6 \cdot 66),(7 \cdot 00)$.
The average interval is about $0.2 \mathrm{MV}$. and the spacing is approximately uniform from one end of the energy scale to the other. There is some evidence for a doublet structure between $1.5 \mathrm{MV}$. and $4 \mathrm{MV}$. We may note that the rapid decrease in intensity with energy is partly due to the highly inhomogeneous distribution of energy of the neutrons from the source, but it is also partly due to the established decrease in the yield from nitrogen at higher neutron energies. The smoothed curve shows a minimum slope at about $3 \mathrm{MV}$.; this fact is discussed later.

Interpretation and Discussion. Perhaps the main result of the work is the interpretation of the thirtytwo peaks as thirty-two energy-levels of ${ }^{15} \mathrm{~N}$. The question then arises whether all the groups consist of the same type of particles; in particular, do the low-energy groups, so affected in appearance by the introduction of paraffin wax, differ in any way from the groups of higher energy?

Before answering this question, Comparat points out comparisons with other workers. Wilhelmy, Maurer and others, found fewer groups with an interval of about 0.5 MV. Comparat claims that he includes these groups and has demonstrated the existence of the others owing to the superior sensitivity and selectivity of his apparatus, the resolving power of which was estimated at about $0.1 \mathrm{MV}$. In support of the reality of these levels he quotes recent work by Hansen which also shows a large number of levels although the source was quite different, the neutrons being obtained by the deuteron bombard. ment of beryllium or lithium.

The particles at lower energy, the production of which is affected by the thickness of paraffin wax, are considered to be protons produced by the reaction

$$
{ }^{14} \mathrm{~N}+{ }^{1} n \rightarrow{ }^{15} \mathrm{~N} \rightarrow{ }^{14} \mathrm{C}+{ }^{1} \mathrm{H}
$$

The particles of higher energy are $\alpha$-particles pro. duced according to reaction ( 1 ). The existence of two groups of particles explains the change in shape of the smoothed curve at about $3 \mathrm{MV}$. The curve is assumed to be compounded of two curves, one giving the yield of protons and the other that of $\alpha$-particles, the latter curve having a maximum in the region of $3 \mathrm{MV}$. Careful examination shows that at very low energies the curve again shows a change of shape and this is attributed to a third type of interaction, namely, the elastic recail of nitrogen nuclei on neutron impact.

Comparat conceives the possibility of an apparatus of higher resolving power demonstrating the existence of still more levels of ${ }^{15} \mathrm{~N}$. That the number of undiscovered levels should prove large he considers improbable on the practical grounds of the reproducibility of existing groups under conditions of varying sensitivity of his apparatus and on theoretical considerations of the spacing of the energy levels of light nuclei. Theory also supports the view that the low-energy particles are protons, since $\alpha$-particles of such low energy would have very great difficulty in penetrating the potential barrier of the nitrogen nucleus.

By extending these experiments to oxygen and other elements a veritable nuclear spectroscopy of energy levels could be evolved. Valuable information. on nuclear structure might then be forthcoming by examining the distribution and width of energy levels as a function of the number of nuclear particles, thus throwing light on the nature of the forces which bind these nuclear particles together.

F. C. Champion. 